\title{
Mind Transmitting via Brainwave's Transforming and Emission
}

\author{
Wang Xinye* \\ Independent Researcher, China
}

*Corresponding author: Wang Xinye, Independent Researcher, China; Email: wang_xinye@ yahoo.com

\section{Research Article \\ Volume 5 Issue 1}

Received Date: March 29, 2021

Published Date: April 06, 2021

DOI: $10.23880 / p s b j-16000168$

\section{Abstract}

The mind is one of activities of human brain from cognitive aspects to noncognitive aspects and displayed by electrical signals of brainwave. The electrical signals can be either converted into infrasound and LF sound with electromechanical transducer and transmitted in the atmosphere or converted into ELF and SLF radio waves by modulation technique and emitted out with transmitter from antenna in Space. The information carrying by the newly generated waves can be received and recorded in another places. The brainwave's pattern of every individual is unique, so the message come from brainwaves can be used for biometric identification.

Keywords: Neural Oscillation; Electrical Impulse and Signal; Brainwave; Mind; Infrasound and LF Sound; ELF and SLF Radio Wave

\section{Introduction}

Hans Berger, the German Psychiatrist is well known as the inventor of electroencephalography (EEG) in 1924, which is used for recording the electrical activity of the brain, commonly described in terms of brain waves $[1,2]$ and as the discover of Alpha wave, named in his honor as Berger Wave [3].

The brainwave or neural oscillation is an electrical impulse in the brain. These brainwaves can be measured in frequency and amplitude (the electrical potential or how many cells fire at the same time and the same speed is expressed as voltage). An individual 's behaviors, emotions and thoughts are communicated between neuromas with electrical impulse in the brain. From fastest to slowest, there are five widely recognized brainwaves, classified from their frequencies and responding to their brain's activities states:

Delta waves $(0.5-4 \mathrm{~Hz})$, belonging to the unconsciousness state are usually associated with the deep stage 3 of NREM
(Non-rapid eye Movement sleep), also known as slow-wave sleep (SWS) and aid in characterizing the depth of sleep. Theta waves $(4-7 \mathrm{~Hz})$ belonging to the subconsciousness state underlie various aspects of cognition and behavior including learning, memory, spatial navigation in many animals.

Alpha waves $(8-12 \mathrm{~Hz})$ belonging to consciousness fading states indicate that the brain is in a resting state. Beta waves $(12.5-30 \mathrm{~Hz})$ are usually divided into three ranges: low range (12.5-16 Hz), relax but cathexis; middle range (16.5$20 \mathrm{~Hz}$ ), think deeply; high range, exciting and anxiety.

Gamma waves $(25-140 \mathrm{~Hz}$, mainly in $40 \mathrm{HZ})$ are the most common pattern in humans, correlated with large scale brain network activity, cognitive phenomena such as working, memory, attention and perceptual can be increased in amplitude via meditation or neurostimulation [4-8].

The brainwave's pattern of every individual is unique, and that the electroencephalogram can be used for biometric 
identification [9]. EEG-based biometry is an emerging research topic and may open a new research direction and application in the future.

\section{The Mind is One of Activities of Human Brain and Correlated with the Electrical Signals of Brainwave}

The mind is the set of faculties including cognitive aspects such as consciousness, imagination, perception, thinking, intelligence, judgement, language, and memory as well as noncognitive aspects such as emotion and instinct. Under the scientific physicalist interpretation, the mind is produced at least in part by the brain [10].

The brain activities can be accurately reflected from the state of the brain wave:

Theta wave represents the subconsciousness state of cognitive aspects, Beta wave represents both on cognitive and on noncognitive aspects. Gamma wave represents cognitive aspect. Although Delta wave belongs to the unconsciousness state and Alpha wave belongs to consciousness fading state, the brain activities do not stop, the brain waves are still exit: Delta wave means sleep state and Alpha wave means Inspiration and instinct [8].

\section{The Mind Transmits via Brainwave's Transforming and Emission}

The mind can be transmitted after the brainwaves are transformed:

The Electrical Signals of Brainwave are Converted into Infrasound and LF Sound Waves and then Transmitting in the Atmosphere: The Delta wave, Theta wave, Alpha wave and Beta wave are with frequencies mostly bellow $20 \mathrm{~Hz}$. If the electrical signals of above brainwave are converted into infrasound wave, the Gamma wave is converted into LF (Low Frequency) sound waves, keeping frequency unchanged and this infrasound and LF sound waves carrying brainwave's information can be transmitting in the atmosphere [11].

The transforming from electrical signal to infrasound and LF sound wave can be completed with Electromechanical transducer, which either convert an electrical signal into sound wave (as in a loudspeaker) or converts a sound wave into an electrical signal (as in a microphone) [12].

The infrasound or LF sound waves that are converted from electrical signals of brainwave will propagate with the velocity as same as sound wave [13].
The newly generated infrasound or LF sound waves from brainwaves possess some special features due to the very low frequency and very high wavelength:

1. The infrasound waves are not easy to decay and to be absorbed by air or water and are able to bypass largescale obstacles by diffraction, so the infrasound waves can propagate very long distance and wide area than sound, light and radio waves. Such as the infrasound wave produced from volcanic eruption of South Sumatra island in 1883 went round the Earth three times, traveled over $100,000 \mathrm{~km}$ and lasted 108 hours [14].

2. The infrasound waves have strong penetrating power. A sound waves with $7000 \mathrm{~Hz}$ can be blocked with a piece of paper while a infrasound wave with $7 \mathrm{~Hz}$ can go through more than ten-meter-thick reinforced concretes.

As discussed above, an infrasound waves converted from brainwaves and carrying brain activity information can bypass large scale obstacles, go through strong barriers and spread far and wide.

The Electrical Signals of Brainwave are Converted into ELF and SLF Radio Wave and then Transmitting in Space: The electrical signals of brainwave are firstly converted into infrasound and LF sound waves, then the infrasound and LF sound waves are superimposed onto a radio carrier wave to produce an ELF (Extremely Low Frequency,3Hz-30Hz) and a SLF (Super Low Frequency,30Hz-300Hz) radio waves with modulation technique. These AM radio waves carrying brainwave's message can be sent out with transmitter from antenna in Space [15].

ELF and SLF radio waves are not easy to decay and can spread far and wide, even underwater [16].

\section{Conclusion}

The mind, one of the brain's activities can be either converted into infrasound and LF sound wave, then transmitted in the atmosphere or converted into ELF and SLF radio wave, then transmitted in Space. These newly generated waves carrying brain activity's information can be received and recorded in another places.

\section{References}

1. Tudor M, Tudor L, Tudor KI (2005) Hans Berge r(18731941)--the history of electroencephalography. Acta Medica Croatica 59(4): 307-313.

2. David Millet (2002) The Origins of EEG. International 


\section{Physical Science \& Biophysics Journal}

Society for the history of the Neurossciences (ISHN), USA.

3. Rumeysa I, Adanir SS, Sevmez F (2020) The inventor of electroencephalography (EEG): Hans Berger(1873-1941). Child's Nervous System.

4. Foster JJ, Sutterer DW, Serences JT, Vogel Ek, Awh E (2017) Alpha- band Oscillations Enable Specially and Temporally Resolved Tracking of Covert Spatial Attention. Psychol Sci 28(7): 929-941.

5. Lutz A, Greischar LL, Rawlings NB, Ricard M, Davidson RJ (2004) Long-term meditator's self-induce highamplitude gamma synchrony during mental practice. Proc Natl Acad Sci USA 101(46): 16369-16373.

6. McDermott E, Porter E, Hughes D, McGinley B, Lang M, et al. (2018) Gamma Band Neural Stimulation in Humans and the Promise Modality to Prevent and Treat Alzheimer's Disease. J Alzheimers Dis 65(2): 363-392.

7. Thomson H (2018) How flashing lights and pink noise might banish Alzheimer 's improve memory and more. Nature 555(7694): 20-22.

8. Po-Chin K, Yi-Ti C, Yong-Sheng C, Li-Fen C (2017) Decoding the Perception of Endogenous Pain from Resting-state MEG. Neuroimage 144(Pt A): 1-11.

9. Marcel S, Millan JDR (2007) Person authentication using brainwaves (EEG) and maximum a posteriori model adaptation. IEEE Trans Pattern Anal March Inteii 29(4): 743-752.

10. Olive Elbs (2005) Neuro-Esthetics: Mapological foundations and applications(Map 2003). Munichen: M Press.

11. Shams QA, Zuckerwar AJ, Knight HK (2014) Development and installation of an infrasonic wake vortex detection system at Newport News International Airport. WakeNet-Europe, France.

12. Berg RE (2018) Electromechanical transducer instrument. Britannica.

13. ELGabry MN, Korrat IM, Hussein HM, Hamama IH (2017) Infrasound detection of meteors. NRIAG Journal of Astronomy and Geophysics 6(1).

14. Brata AG, Rietveld P, de Groot HLF, Zant W (2013) The Krakatau eruption in 1883: Its Implications for the Spatial Distribution of Population in Java. Economic History of Developing Regions 28(2): 27-55.

15. Ellingson SW (2016) Radio Systems Engineering. Cambridge University Press, USA.

16. Wolkoff EA (1992) ELF/VLF/LF Radio Propagation and System Aspects. AGARD Conference Proceedings, Elemmagnetic Wave Propagation Panel Symposium, Belgium. 